\title{
ANÁLISE DA DINÂMICA DE FRAGMENTOS FLORESTAIS NO MUNICÍPIO DE CARANDAÍ, MG, PARA FINS DE RESTAURAÇÃO FLORESTAL ${ }^{1}$
}

\author{
Leandro Calegari², Sebastião Venâncio Martins ${ }^{3}$, José Marinaldo Gleriani ${ }^{3}$, Elias Silva ${ }^{3}$ e Luiz Carlos Busato ${ }^{4}$
}

\begin{abstract}
RESUMO - A busca de conhecimento sobre fragmentos florestais nas propriedades rurais permite a aplicação de uma correta gestão agroambiental, favorecendo a sua conservação e restauração em áreas degradadas. Utilizando os softwares SPRING e FRAGSTATS, caracterizou-se a paisagem, com ênfase nos fragmentos de Floresta Estacional Semidecidual Secundária, localizada no Município de Carandaí, MG, e região de entorno, considerando sua evolução durante o período de 1984 a 2007. Concluiu-se que ocorreu aumento de quase $8 \%$ na área total de floresta nativa devido ao surgimento de 46 novos fragmentos, e a área individual de cada fragmento apresentou tendência de redução (31,9 para 30,6 ha), o que proporcionou aumento da densidade de borda (17,1 para 19,7 m/ha), diminuindo a porcentagem de cada fragmento, que é a área central (33,8 para 30,2\%) durante o intervalo de tempo avaliado. Apesar da vantagem de predominância de fragmentos de forma geométrica simples (índice de forma médio de 1,738), com redução do efeito de borda, a qualidade da paisagem apresentou-se comprometida, por ser constituída predominantemente por pequenos fragmentos. Os fragmentos florestais mostraram-se ainda muito distanciados entre si (mais que $200 \mathrm{~m}$ ), apresentando pequena tendência de aglomeração (reflexo do aumento no número de fragmentos), porém ainda insuficiente. Portanto, apesar de a cobertura de floresta nativa ter apresentado aumento de área total, de modo geral esta perdeu em qualidade no intervalo de tempo estudado. Essas informações podem ser utilizadas para uma gestão ambiental correta quanto ao manejo florestal nessa região.
\end{abstract}

Palavras-chave: Ecologia da Paisagem, Remanescentes florestais, Sistemas de Informações Geográficas e Biologia da Conservação.

\section{ANALYSIS OF THE DYNAMICS OF FOREST FRAGMENTS IN THE CITY OF CARANDAÍ, MG, FOR FOREST RESTORATION}

\begin{abstract}
The knowledge on forest patches in rural properties allows the application of a correct environmental administration, promoting their conservation and restoration in degraded areas. By using the softwares SPRING and FRAGSTATS, the landscape was characterized with emphasis on the patches of secondary Seasonal Semidecidual Forest, located in the municipal district of Carandaí, MG, and the area around it, considering its evolution from the period of 1984 to 2007. It could be concluded that progressive increase in the total area of native forest occurred due to the appearance of new patches, and the individual area of each patch presented reduction tendency, what provided increase of the edge area, reducing the percentage of each patch which is the core area during the gap of the evaluated time. Despite the advantage of predominance of patches in simple geometric ways, with reduction of the edge effect, the quality of the landscape got threatened because it was predominantly constituted by small patches. The forest patches were still so distant one from the others (more than 200 meters), presenting small gathering tendency (result of the increase of the number of patches), however, still insufficient. Therefore, although the covering of native forest has presented increase of total area, in general, it lost in quality in the gap of the time considered.
\end{abstract}

Keywords: Landscape Ecology, Forest patches, Geographical Information System and Conservation Biology.

\footnotetext{
${ }^{1}$ Recebido em 03.05.2009 e aceito para publicação em 02.03.2010.

${ }^{2}$ Universidade Federal de Campina Grande, UFCG, Brasil. E-mail: <calegari@cstr.ufcg.edu.br>.

${ }^{3}$ Universidade Federal de Viçosa, UFV, Brasil. E-mail: <venancio@ufv.br>.

${ }^{4}$ Signus Vitae - Projetos Ambientais Inteligentes e Cimento Tupi S/A, Volta Redonda, RJ. E-mail: <lcb@signusvitae.com.br>.
} 


\section{INTRODUÇÃO}

O processo de fragmentação florestal que se intensifica nos tempos recentes tem resultado na formação de paisagens com pouca diversidade de hábitat, com fragmentos isolados e de dimensões reduzidas.

Um fragmento florestal pode ser definido como uma área de vegetação natural interrompida por barreiras antrópicas ou naturais (ex.: estradas, povoados, culturas agrícolas e florestais, pastagens, montanhas, lagos, represas) capazes de diminuir significativamente o fluxo de animais, pólen e, ou, sementes (VIANA, 1990). Como principais consequências da fragmentação provocada por ação abiótica, podem-se citar as alterações no microclima como na umidade do ar, temperatura e radiação solar, particularmente nas bordas dos fragmentos, que ficam mais sujeitas a exposição solar. Outra ação abiótica é o aumento dos riscos de erosão, assoreamento dos cursos d’água e redução gradativa dos recursos hídricos, pela menor capacidade de retenção de água das chuvas nos solos, maior velocidade de escoamento destas e também maior evapotranspiração. Entre as consequências da fragmentação de origem biótica, podem-se citar a perda da biodiversidade microbiológica do solo, da flora e da fauna, a perda da diversidade genética, a redução da densidade ou abundância, alteração da estrutura da vegetação e maior possibilidade de ocorrência de espécies invasoras, entre outros. Esses danos podem ocorrer para a espécie em particular ou para a comunidade de plantas, podendo ainda provocar a modificação ou, mesmo, a eliminação das relações ecológicas originalmente entre as espécies vegetais, os polinizadores e os dispersores (BORGES et al., 2004).

A busca de conhecimento ecológico sobre os fragmentos florestais de uma área permite aplicar a gestão ambiental correta quanto ao manejo florestal, este podendo ser estudado pela Ecologia da Paisagem, através da cartografia de uso e cobertura das terras. Dessa forma, a avaliação da paisagem torna-se importante para diagnosticar os problemas atuais, estimar influências futuras e apontar as mudanças necessárias para manter o equilíbrio natural.

O uso integrado dos Sistemas de Informações Geográficas (SIGs) e imagens de satélite de alta resolução é de extrema importância em estudos de Ecologia da Paisagem, por permitirem a detecção precisa dos fragmentos remanescentes, seus estados de conservação e as áreas em que o uso da terra se encontra conflitante com a legislação ambiental vigente (BRITO et al., 2007; OLIVEIRA et al., 2008).

Nesse sentido, este estudo buscou caracterizar a paisagem, com ênfase nos fragmentos de Floresta Estacional Semidecidual secundária, localizada na região envolvendo o Município de Carandaí, MG considerando-se sua evolução durante o período de 1984 a 2007.

\section{MATERIAL E MÉTODOS}

Este estudo faz parte de um projeto maior que visa à restauração florestal de uma propriedade rural de aproximadamente 100 ha localizada no Município de Carandaí, MG, pertencente à empresa Cimento Tupi S.A.

Foram utilizadas imagens disponibilizadas pelo Instituto Nacional de Pesquisas Espaciais (INPE) do sensor TM/Landsat 5 (1984, 1994 e 2007) e imagem do sensor ETM+/Landsat 7 (2001) do portal Land Cover Facility da órbita/ponto 218/74. A cena de 2001 (UTM/ WGS-84) foi utilizada como referência para o georreferenciamento das demais cenas. Baseou-se em pelo menos 10 pontos de controle para obter uma raiz do erro médio quadrático (RMSE) menor que 0,5 pixel para os pontos de teste. A cena do sensor ETM+/ Landsat-7 foi reamostrada para $30 \mathrm{~m}$, uma vez que estava disponibilizada em $28,5 \mathrm{~m}$. Todas as métricas expressas neste trabalho estão nessa resolução, em um contexto de 807 km², uma vez que variações nos valores das métricas podem ocorrer pela natureza dos dados (matricial) e pela extensão analisada (WICKHAM e RITTERS, 1995; TURNER et al., 1989).

Na primeira fase do trabalho, utilizou-se o software SPRING 4.3. Gerou-se uma imagem para cada ano (1984, 1994, 2001 e 2007) na composição colorida 4R5G3B, ou seja, as bandas 4 , 5 e 3 do sensor TM/Landsat5 nas cores vermelha (Red), verde (Green) e azul (Blue).

Uma vez que a paisagem deve ser estudada no seu contexto e fragmentos florestais vizinhos a determinada área de estudo também influenciam os fatores ecológicos no interior dessa área, foi realizado um recorte das imagens resultantes, tendo a área em processo de restauração (Município de Carandaí, MG) como foco inicial e englobando ainda municípios vizinhos, como Casa Grande, Cristiano Otoni e Caranaíba. Dessa forma, a área de estudo (paisagem) correspondeu ao quadrante formado entre as coordenadas $20^{\circ} 43^{\prime} 32^{\prime \prime S}$ /

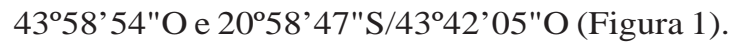


Fonte: Adaptado de GOOGLE EARTH, 2008. Source: Adapted from GOOGLE EARTH, 2008.

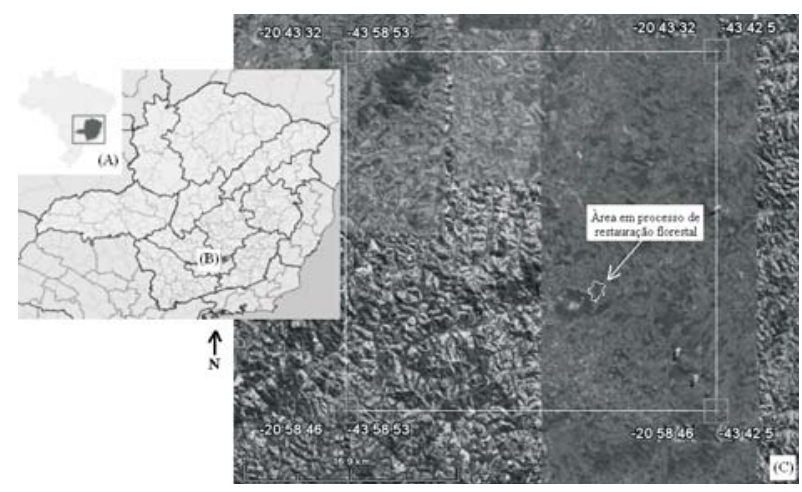

Figura 1 - Localização do Estado de Minas Gerais no Brasil (A), Município de Carandaí no Estado (B) e quadrante correspondente à área de estudo (C).

Figure 1-Location of the state of Minas Gerais in Brazil(A), municipal district of Carandaí in this state (B) and quadrant corresponding to the area being studied (C).
Devido ao fato de a classificação digital não se mostrar eficaz, principalmente em razão do sombreamento resultante do relevo, realizou-se a interpretação visual, digitalizando a classe floresta nativa (Floresta Estacional Semidecidual Secundária). Essa classe se destacava das demais pela textura rugosa e pela cor vermelha.

Após a associação da classe, a imagem vetorial foi convertida em matricial ( $30 \mathrm{~m}$ ) e exportada no formato ASCII, que é um dos formatos reconhecidos pelo software FRAGSTATS 3.3 (MCGARIGAL et al., 2002).

O FRAGSTATS calcula mais de 50 métricas, sendo muitas delas redundantes para determinadas situações (MCGARIGAL et al., 2002). Para quantificar a estrutura e composição dos fragmentos florestais e comparálas numa série histórica (1984, 1994, 2001 e 2007), utilizaram-se parâmetros em nível de classe de fragmentos e paisagem (Tabela 1).

Tabela 1 - Índices de Ecologia da Paisagem gerados pelo software FRAGSTATS (versão 3.3) para quantificação da estrutura da paisagem da classe Floresta Estacional Semidecidual Secundária. Consultar McGarigal et al. (2002) para a descrição completa e fórmula de cada índice.

Table 1 - Indexes of Landscape Ecology generated by the software FRAGSTATS (version 3.3) for quantification of the landscape structure of secondary Seasonal Semidecidual Forest. Check McGarigal et al. (2002) for complete description and formula for each index.

\begin{tabular}{|c|c|c|c|}
\hline Métrica & $\begin{array}{l}\text { Sigla e intervalo } \\
\text { (unidade) }\end{array}$ & Observação & Conseqüências \\
\hline Área da paisagem & TA $>0$ (ha) & Área total da paisagem & $\begin{array}{c}\text { Utilizada principalmente } \\
\text { nos cálculos dos demais } \\
\text { índices }\end{array}$ \\
\hline Área da classe & CA $>0$ (ha) & $\begin{array}{c}\text { Somatório da área } \\
\text { dos fragmentos da classe }\end{array}$ & $\begin{array}{c}\text { Maior valor favorece para a } \\
\text { conservação, correspondendo } \\
\text { a maior cobertura da paisagem } \\
\text { paisagem pela classe }\end{array}$ \\
\hline $\begin{array}{l}\text { Índice do maior } \\
\text { fragmento }\end{array}$ & $0<\mathrm{LPI} \leq 100(\%)$ & $\begin{array}{l}\text { Percentagem da paisagem } \\
\text { paisagem ocupada pelo }\end{array}$ & $\begin{array}{l}\text { Maior valor favorece de } \\
\text { dispersores, polinizadores } \\
\text { e propágulos, abastecendo } \\
\text { fragmentos menores }\end{array}$ \\
\hline $\begin{array}{l}\text { Número de } \\
\text { fragmentos }\end{array}$ & $\begin{array}{l}\mathrm{NP} \geq 1 \\
\text { (adimensional) }\end{array}$ & $\begin{array}{l}\text { Número total de } \\
\text { fragmentos da classe }\end{array}$ & $\begin{array}{l}\text { Maior valor indica maior } \\
\text { fragmentação da paisagem }\end{array}$ \\
\hline $\begin{array}{l}\text { Área média dos } \\
\text { fragmentos }\end{array}$ & $\underset{\text { (ha) }}{\operatorname{AREA} M N}>0$ & $\begin{array}{l}\text { Área média de todos os } \\
\text { fragmentos da classe }\end{array}$ & $\begin{array}{l}\text { Fragmentos de tamanhos } \\
\text { maiores apresentam } \\
\text { maior diversidade, uma } \\
\text { vez que a riqueza das } \\
\text { espécies depende das } \\
\text { dimensões dos fragmentos }\end{array}$ \\
\hline $\begin{array}{l}\text { Densidade de } \\
\text { bordas }\end{array}$ & $\underset{(\mathrm{m} / \mathrm{ha})}{\mathrm{ED}} \geq 0$ & $\begin{array}{l}\text { Comprimento total de } \\
\text { bordas dividido pela área } \\
\text { total da paisagem }\end{array}$ & $\begin{array}{c}\text { Maior valor implica em maior } \\
\text { efeito de borda, portanto, } \\
\text { maléfica }\end{array}$ \\
\hline
\end{tabular}

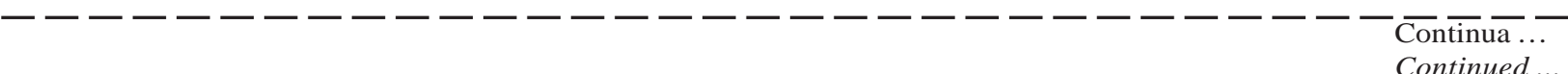


Tabela 1 - Cont.

Table 1 - Cont.

\begin{tabular}{|c|c|c|}
\hline $\begin{array}{l}\text { Índice de forma } \\
\text { médio }\end{array}$ & $\begin{array}{l}\mathrm{SHAPE} \text { A MN } \geq 1 \\
\text { (adimensional) }\end{array}$ & $\begin{array}{l}\text { Valor médio do índice de } \\
\text { forma dos fragmentos da } \\
\text { classe }\end{array}$ \\
\hline Área central total & $\mathrm{TCA} \geq 0$ & $\begin{array}{l}\text { Soma das áreas centrais } \\
\text { toda a classe (borda }=60\end{array}$ \\
\hline
\end{tabular}

Número de áreas centrais

NDCA $\geq 0$ (adimensional)

$$
\begin{gathered}
0 \leq \mathrm{CAI}_{(\%)} \mathrm{MN} \leq 100 \\
\underset{(\mathrm{ENN} / \mathrm{MN}}{\mathrm{EN}} \geq 0 \\
0 \leq \underset{\text { AREA_CV } \leq}{100(\%)} \\
0 \leq \text { ENN_CV } \leq 100 \\
(\%)
\end{gathered}
$$

variação da

distância do vizinho
Número total de áreas centrais disjuntas da referida classe

Percentagem média da classe que é área central

Valor médio do vizinho mais próximo dos fragmentos da classe

Variabilidade do tamanho médio dos fragmentos da classe

Variabilidade da

distância média do vizinho mais próximo da classe
Valor menor indica fragmento de forma simples, benéfico para a conservação

Valor maior indica melhor qualidade da paisagem, uma vez que corresponde a área total do habitat preservado dentro dos fragmentos

Apesar de corresponder a área preservada dentro dos fragmentos, seu aumento significa o aumento da fragmentação florestal

Valor menor indica maior efeito de borda, maléfico à conservação

Valor menor favorece para a aglutinação dos fragmentos, favorecendo ao fluxo gênico Alto valor indica alta heterogeneidade espacial da paisagem, favorecendo a diversidade

Alto valor indica alta variabilidade para distâncias entre fragmentos, prejudicando o fluxo gênico
Utilizou-se uma distância de 60 m para a largura de borda. A seleção das métricas e a largura da borda basearam-se nos estudos de Waldhoff e Viana (1993), Albanez (2000), Pereira et al. (2001), Costa (2003), Borges et al. (2004) e Fushita (2006).

A única métrica em nível de paisagem empregada (TA) foi utilizada para a determinação do tamanho total da paisagem. As métricas restantes (nível de classe) foram utilizadas para a geração de gráficos no Excel, a fim de serem mais bem comparadas.

\section{RESULTADOS E DISCUSSÃO}

A área total da paisagem (TA), que se refere ao quadrante apresentado na Figura 1, foi de 80.770,5 ha para todos os anos analisados, uma vez que corresponde à mesma paisagem. As métricas analisadas em nível de classe são apresentadas nas Figuras 2 e 3 .

A evolução histórica da paisagem indicou progressivo aumento na área total ocupada pela classe (CA). Esse acréscimo de 926 ha pode ser possivelmente explicado pelo abandono das áreas rurais, bem como pela atual restrição imposta pela legislação ambiental.
Entretanto, da mesma forma que o observado por Albanez (2000) e Greggio et al. (2009), nos Municípios de Ponte Nova, MG, e Jaboticabal, SP, respectivamente, a área total ocupada pela classe ainda encontra-se aquém da exigida pelo Código Florestal (20\%), que neste estudo correspondeu a $16 \%$ da área total da paisagem no ano de 2007 (Figura 2A).

Conforme Costa (2003), apesar de o Código Florestal ter evitado a retirada total, favoreceu a fragmentação da vegetação natural das diversas propriedades rurais, à medida que priorizava a proteção das áreas de proteção permanente e de reserva legal.

O maior fragmento florestal, denominado matriz, apresentava uma área aproximada de 1.442 ha no ano de 1984 (índice do maior fragmento-LPI = 1,785); mostrou grande redução até 1994, permanecendo posteriormente constante até 2007 , quando se encontrava com cerca de 1.257 ha (LPI = 1,556). Portanto, ocorreu clara redução da área desse importante fragmento (Figura 2B), comportamento semelhante ao descrito por Tonial (2003), ao analisar bacias hidrográficas na região Noroeste do Rio Grande do Sul. 
O conceito de que a dinâmica de população pode depender da qualidade relativa de hábitats (bons e ruins) é chamado de dinâmica fonte-sumidouro. Esse modelo considera o movimento dos indivíduos entre os fragmentos para a manutenção de populações, em que a taxa de nascimentos excede a taxa de morte em um ou mais hábitats denominados fontes, mas a taxa de morte excede a taxa de nascimento em um ou mais hábitats denominados sumidouros. As populações localizadas em áreas consideradas como sumidouros ocupam manchas de hábitats de baixa qualidade que não as suportam por longo tempo,
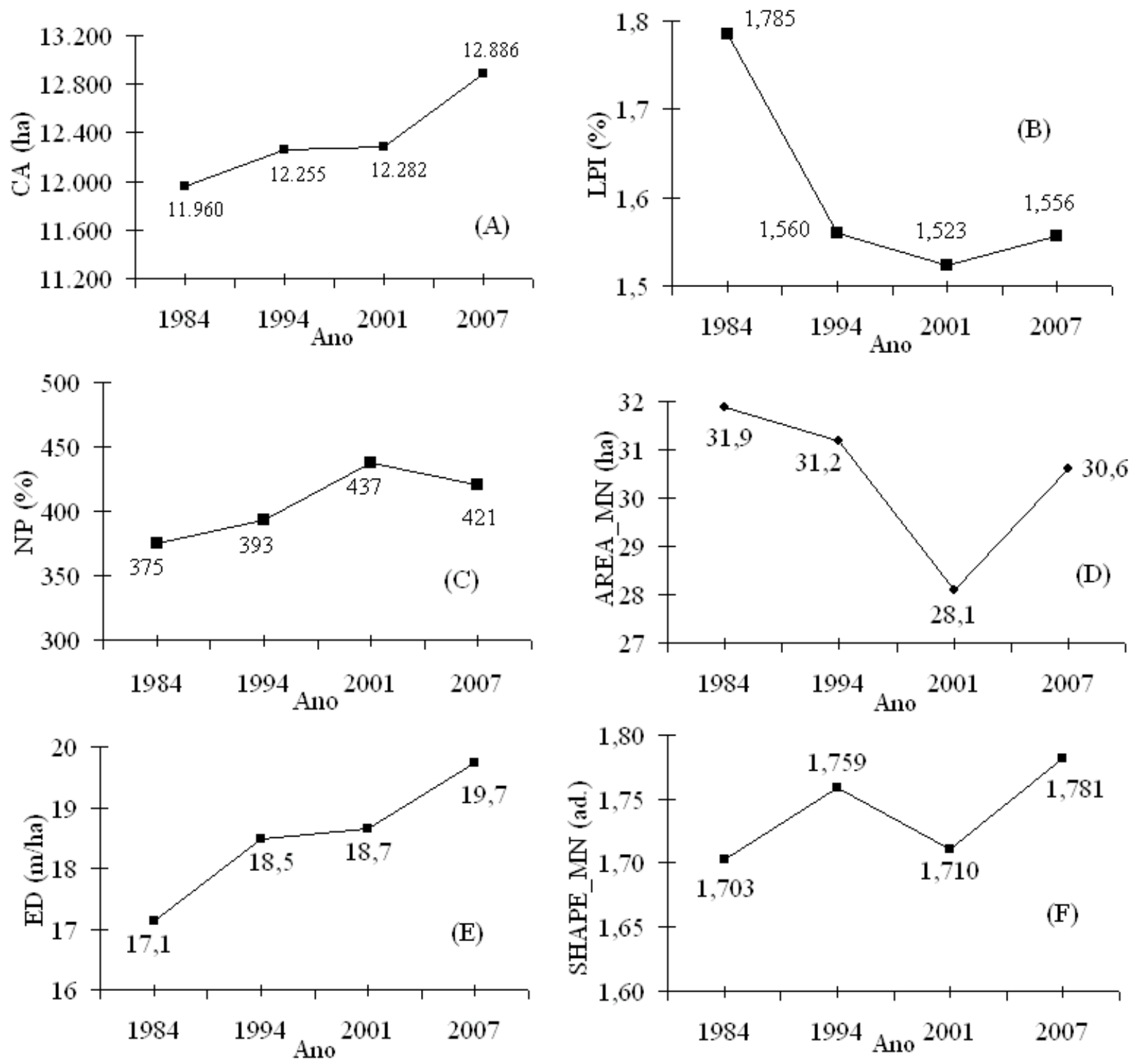

Figura 2 - Métricas da classe floresta nativa (Floresta Estacional Semidecidual Secundária), Município de Carandaí, MG: A) área total (CA); B) índice do maior fragmento (LPI); C) número de fragmentos (NP); D) tamanho médio dos fragmentos (AREA_MN); E) densidade de bordas (ED); e F) índice de forma médio (SHAPE_MN).

Figure 2 - Metrics of the native forest class (secondary Seasonal Semidecidual Forest), municipal district of Carandaí, MG: A) total class area (CA); B) largest patch index (LPI);C) number of patches (NP); D) mean patch area (AREA_MN); E) edge density (ED); F) mean shape index (SHAPE_MN). 
e as populações em áreas consideradas como fonte, em um hábitat de alta qualidade, resistem por mais tempo e contribuem para a recolonização de outros fragmentos, por meio da dispersão de indivíduos (FUSHITA, 2006). Dessa forma, pode-se entender a importância do fragmento-matriz, por ser importante área-fonte de dispersores, polinizadores e propágulos, criando condições favoráveis para a manutenção da biodiversidade local, por meio de processos-chave, como a dispersão, polinização etc.
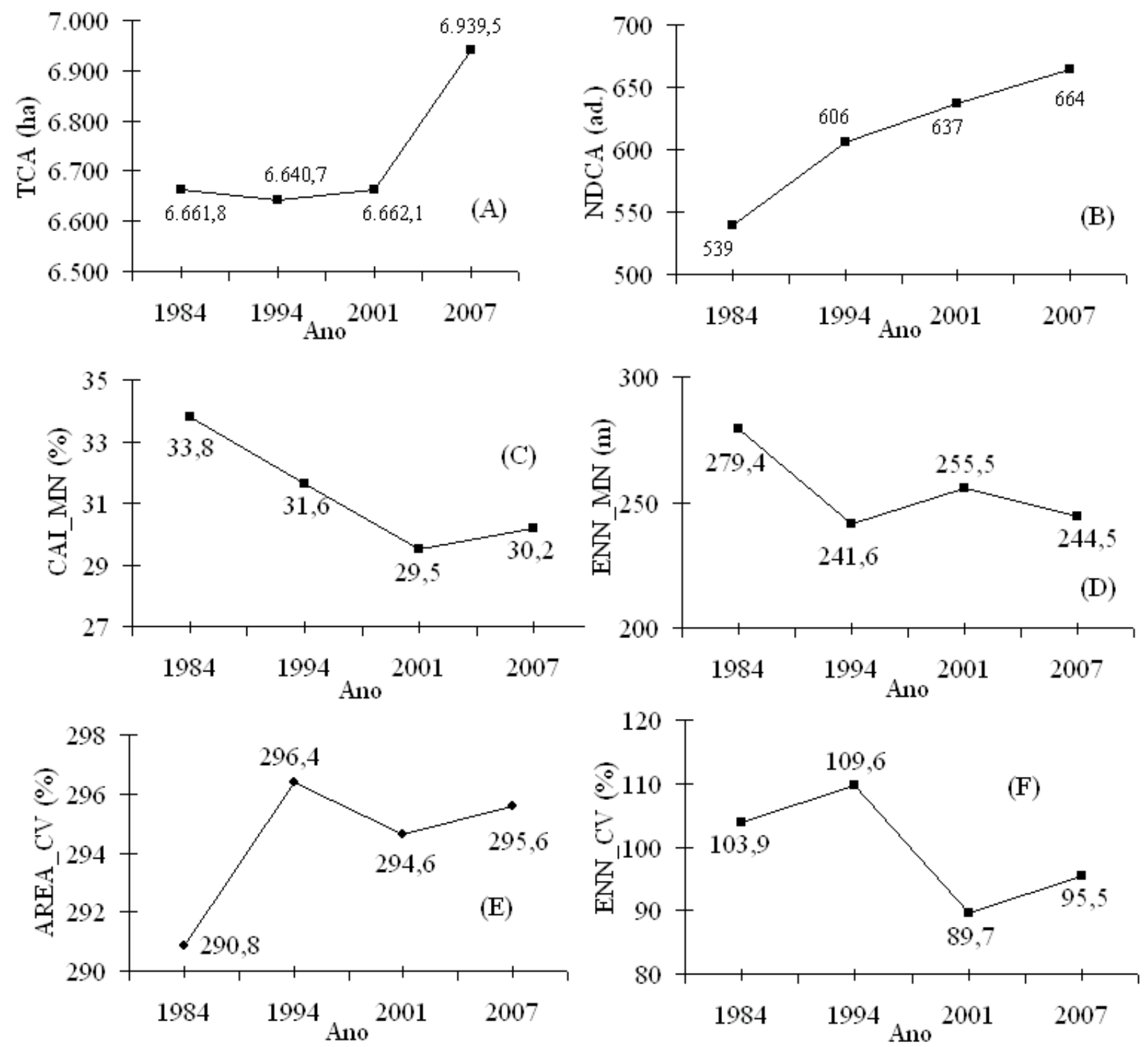

Figura 3 - Métricas da classe floresta nativa (Floresta Estacional Semidecidual Secundária), Município de Carandaí, MG: A) área central total (TCA); B) número de área central (NDCA); C) índice de área central média (CAI_MN); D) distância média do vizinho mais próximo (ENN_MN); E) coeficiente de variação do tamanho médio dos fragmentos (AREA_CV); e F) coeficiente de variação da distância média do vizinho mais próximo (ENN_CV).

Figure 3 - Metrics of the native forest class (secondary Seasonal Semidecidual Forest), municipal district of Carandaí, MG: A) total core area (TCA); B) number of disjunct core areas (NDCA); C) mean core area index (CAI_MN); D) mean Euclidean nearest-neighbor distance; (ENN_MN); E) coefficient of variation patch area (AREA_CV); F) coefficient of variation Euclidean nearest-neighbor distance (ENN_CV).

Revista Árvore, Viçosa-MG, v.34, n.5, p.871-880, 2010 
O aumento na área total ocupada pela classe (CA) ocorreu devido ao crescimento do número de fragmentos (NP) (Figura 2C), uma vez que se observou tendência de redução do tamanho médio dessas áreas (AREA_MN) (Figura 2D). Portanto, teve-se uma adição de 46 novos fragmentos florestais, com seus tamanhos médios passando de 31,9 ha para 30,6 ha durante o período estudado. De maneira contrária, Greggio et al. (2009) observaram diminuição considerável na área de mata num período de 29 anos (1971 a 2000), no Município de Jaboticabal, SP. Esse comportamento foi explicado pela redução da área dos fragmentos, e a quantidade deles manteve-se praticamente igual. O aumento da área de determinada classe pode ocorrer também devido ao incremento do tamanho dos fragmentos, de forma mais marcante do que o surgimento de novos, conforme constatado por Pereira et al. (2001).

O número de fragmentos de determinada classe é de fundamental importância para os aspectos dos padrões da paisagem, uma vez que constitui uma medida do seu grau de subdivisão ou fragmentação. O tamanho médio dos fragmentos também é considerado bom indicativo do grau de fragmentação, por ser função do número de fragmentos e da área total ocupada pela classe. Dessa forma, paisagens que apresentam menores valores para tamanho médio de fragmento devem ser consideradas como mais fragmentadas (MCGARIGAL et al., 2002).

A ocorrência de grande quantidade de pequenos fragmentos florestais é comum em paisagens de Floresta Atlântica. Ranta et al. (1998), em uma área de Floresta Atlântica, observaram que $48 \%$ dos fragmentos apresentaram área menor que 10 ha, e somente $7 \%$ desses possuíam área maior que 100 ha. Do número total de fragmentos florestais observados no Parque Nacional dos Campos Gerais, PR, Almeida (2008) observou que 91,4\% tinham áreas inferiores a 5 ha. Albanez (2000), referindo-se ao Município de Ponte Nova, MG, citou que os fragmentos isolados na paisagem, em sua maioria, são constituídos por mata, predominando nas classes de tamanho de 0 a 20 ha, que são considerados pequenos.

Nos pequenos fragmentos florestais, as populações de plantas, principalmente árvores, são constituídas por poucos indivíduos da mesma espécie, gerando percentual considerável de endogamia (cruzamento entre parentes) e alta probabilidade de extinção das espécies no local (COSTA, 2003). Entretanto, esses pequenos remanescentes podem funcionar como stepping stones (pontos de ligação ou trampolins ecológicos), que são pequenas áreas de hábitat dispersas pela matriz que podem, para algumas espécies, facilitar os fluxos entre fragmentos. Promovem aumento no nível de heterogeneidade da matriz e atuam como refúgio para espécies que requerem ambientes particulares que só ocorrem nessas áreas (ALMEIDA, 2008). Portanto, fragmentos pequenos, principalmente quando próximos dos grandes núcleos de biodiversidade, cumprem funções relevantes ao longo da paisagem. Em longo prazo, podem expandir-se, tornando-se ainda mais importantes.

O aumento da densidade de borda (ED), que passou de 17,1 para 19,7 m/ha, também comprova o incremento da fragmentação florestal no intervalo de tempo avaliado (Figura 2E). Quanto maior o número de fragmento de determinada classe, maior a densidade de borda (comprimento total de borda por hectare). Assim, é natural entender que, quanto maior esse valor, maior a fragmentação da paisagem. Aumento da densidade de borda também foi observado por Tonial (2003), o qual citou valores de 55,7; 50,0; 56,0; e 57,2 m/ha nos anos de 1984, 1989, 1994 e 1999, respectivamente.

Na região Centro-Leste do Pará, Pereira et al. (2001) observaram crescimento significativo da densidade de borda de 1973 para 1997, passando de 3 para 25 $\mathrm{m} /$ ha para a paisagem (classes floresta, pasto e capoeira), o que atribuíram ao aumento do número de fragmentos. O aumento da densidade de bordas da classe floresta implicou maior degradação desse ambiente, associada aos maléficos efeitos de borda.

Os valores de índice de forma médio (SHAPE_MN) mostram que a paisagem deste estudo apresenta fragmentos florestais de formas próximas às simples, com pequena tendência de tornarem-se irregulares, o que é evidenciado pelo pequeno aumento dos valores dessa métrica durante o período avaliado: variação de 0,078 nos 23 anos (Figura 2F).

Tonial (2003) observou tendência de aumento da forma de fragmentos florestais com a progressão dos anos, com os fragmentos apresentando formas mais complexas que o observado neste trabalho, sendo os valores de índice de forma médio citados para os anos de 1984, 1989, 1994 e 1999 de 2,12; 2,12; 2,24; e 2,40, respectivamente, com aumento do índice de 0,28 em 15 anos. Variação essa, portanto, superior à verificada neste estudo. Diagnóstico de fragmentos florestais

Revista Árvore, Viçosa-MG, v.34, n.5, p.871-880, 2010 
realizado na bacia hidrográfica do rio Alegre, ES, indicou que a maioria deles possuía forma irregular, o que proporcionava elevado nível de perturbação, uma vez que se tem aumento da área sujeita ao efeito de borda (NASCIMENTO et al., 2006).

A área central, nuclear ou core de um fragmento é o melhor indicativo da qualidade dos fragmentos do que sua área total (MCGARIGAL et al., 2002). A área total central (TCA), que representa a soma das áreas centrais (excluídas as bordas) de toda a classe, manteve-se aproximadamente constante entre os anos de 1984 e 2001 (média de 6.655 ha), apresentando posterior aumento, atingindo 6.939,5 ha em 2007 (Figura 3A), possivelmente devido ao aumento do número de área central (NDCA) (Figura 3B).

O índice de área central média (CAI_MN) representa a percentagem média do fragmento da classe que é a área central. Fragmento com CAI = 0 não possui área central. Essa métrica apresentou tendência de redução dentro do período avaliado (de 33,8 para 30,2\%), indicando que, com o passar dos anos, a menor porcentagem dos fragmentos são áreas centrais, ou maior percentagem dos fragmentos são áreas de bordas (Figura 3C). A redução do valor dessa métrica comprova, mais uma vez, que o aumento da área central total (TCA) deu-se apenas devido ao aumento do número de área central (NDCA), significando redução da qualidade de cada fragmento, da mesma forma como observaram por Périco e Cemin (2006), no Município de Arvorezinha, RS.

Em geral, quanto maior a área total do fragmento, maior a sua área central. No entanto, quando ocorre alto índice de forma, fragmentos de mesmo tamanho total podem apresentar grande diferença de área nuclear. Esse não é o caso deste estudo, uma vez que o índice de forma é baixo (SHAPE_MN médio de 1,738), indicando que a reduzida área nuclear é consequência do pequeno tamanho dos fragmentos.

A distância média do vizinho mais próximo (ENN_MN) apresentou tendência de redução durante o período estudado, que passou de 279,4 para 244,5 m (Figura 3D), consequência do aumento do número de fragmentos (NP), o que faz com que os fragmentos se tornem mais aglutinados.

Tonial (2003) citou valores de ENN_MN iguais a 121, 128, 120 e $119 \mathrm{~m}$ para os anos de 1984, 1989, 1994 e 1999, respectivamente, indicando a tendência de redução do vizinho mais próximo, da mesma maneira que o observado neste trabalho.
Almeida (2008) classificou as distâncias de 60, 120, 200 e >200 m como de baixo, médio, alto e muito alto isolamento, respectivamente. Portanto, de acordo com essa classificação, a paisagem deste estudo demonstrou isolamento muito alto, com grande distância entre os fragmentos de florestas nativas e, por conseguinte, menor capacidade de colonização de espécies em direção a outros fragmentos florestais, prejudicando o fluxo gênico entre populações de animais e vegetais. Essa mesma autora considerou o isolamento dos fragmentos florestais do Parque Nacional dos Campos Gerais, PR, “não muito isolados”, por estarem a distâncias menores de 100 m, defendendo que essa distância é pouco expressiva para alguns grupos de plantas que têm a dispersão feita por mamíferos de pequeno e médio portes. Porém, pode ser considerada uma distância limitante para a movimentação de algumas espécies. Awade e Metzger (2008), por exemplo, observaram que algumas espécies de aves de sub-bosque evitam cruzar áreas abertas com distâncias superiores a $40 \mathrm{~m}$.

Enquanto o coeficiente de variação do tamanho médio (AREA_CV) dos fragmentos tendeu a aumentar (de 290,8 para 295,6\%), o coeficiente de variação da distância média do vizinho mais próximo (ENN_CV) tendeu a diminuir (de 103,9 para 95,5\%) durante o período avaliado (Figura 3E e 3F). Alta variabilidade é comum em estudos de ecologia da paisagem. Basile (2006) e Almeida (2008) também observaram elevados coeficientes de variação em relação à área média dos fragmentos (161 e 705\%, respectivamente), indicando alta heterogeneidade espacial da paisagem. Altos coeficientes de variação do vizinho mais próximo (ENN_CV) indicam alta variabilidade para distâncias entre fragmentos. Analisando áreas de floresta nativa nos Municípios de Bugre e Ipaba, MG, Amaral (2007) verificou fragmentos mais isolados no ano de 1980 (165 m), com CV de aproximadamente 125\%. Esse número diminuiu significantemente no ano de 2004 (16 m), atingindo CV de aproximadamente $178 \%$. Justificou a redução da distância entre os fragmentos devido à implantação de talhões de eucaliptos, o que favoreceu o desenvolvimento da vegetação nativa. A distância até o vizinho mais próximo calculado por Basile (2006) apresentou valor médio de 262 m, também com variação significantemente alta $(\mathrm{CV} \approx 156 \%)$. 


\section{CONCLUSÃO}

Diante das análises obtidas por meio das métricas da paisagem, as quais indicaram a perda da qualidade dos fragmentos florestais na região de Carandaí, MG, durante o período de 1984 a 2007, algumas medidas devem ser tomadas para a melhoria da qualidade ambiental dos fragmentos da região estudada.

As primeiras medidas referem-se à eliminação dos agentes de perturbação. Nas propriedades que possuem gado, deve ser realizado o cercamento dos fragmentos florestais. O fogo, grave problema para a manutenção da biodiversidade, deve ser combatido pela manutenção e, ou, construção de aceiros. A formação de cortinas de proteção e o fomento de pequenos plantios florestais com espécies de rápido crescimento (nativa ou exótica) diminuirão as pressões nos fragmentos nativos remanescentes. Essa formação pode, ainda, diminuir os efeitos de borda e a contaminação química e biológica dos fragmentos, devido às atividades agrícolas do entorno.

Uma vez que ocorre predomínio de fragmentos pequenos na paisagem, estes devem ser unidos para a formação de fragmentos maiores a partir da recomposição da vegetação, o que promoverá o aumentando das áreas centrais. A formação de ilhas e corredores de vegetação unindo fragmentos, principalmente nas propriedades que não possuem reserva legal, pode aumentar, consideravelmente, a proximidade e conectividade entre fragmentos, que até o momento se encontram comprometidos. Deve ser realizada restauração florestal principalmente nas áreas de preservação permanente.

Nas ações de restauração florestal, é preciso trabalhar preferencialmente com a promoção da regeneração natural e, onde essa técnica não for possível, com a regeneração artificial a partir da semeadura direta ou da sucessão secundária induzida. Deverão ser utilizadas as espécies apontadas no levantamento florístico da área-fonte (fragmento de maior tamanho observado na paisagem). O emprego de técnicas de nucleação como a transposição de serapilheira, chuva de sementes, instalação de poleiros e abrigos para a fauna irá aumentar a diversidade de plantas e animais nos fragmentos florestais perturbados.

Nos poucos fragmentos que possuem formas complexas, pode-se realizar a recomposição da vegetação no entorno para a formação de áreas circulares onde o efeito de borda é menor. Isso também favorecerá o aumento das áreas centrais.
Apesar de as imagens do satélite Landsat se mostrarem suficientes para detectar as alterações da paisagem, estas, por serem consideradas de média resolução (pixel de $900 \mathrm{~m}^{2}$ ), oferecem informações limitadas para medidas exatas de áreas, sobretudo de fragmentos florestais pequenos, comuns na região de estudo. Assim, sugerem-se estudos adicionais utilizando o índice de erro Kappa e imagens de outros sensores, tal como do Ikonos II (pixel de $1 \mathrm{~m}^{2}$ ), a fim de comparar a confiabilidade das imagens Landsat para estudos relacionados à ecologia da paisagem.

\section{AGRADECIMENTOS}

Os autores agradecem à Cimento Tupi S/A pelo financiamento do projeto, ao CNPq pela bolsa de produtividade em Pesquisa de S.V. Martins e E. Silva e à Fapemig pela bolsa de Doutorado de L. Calegari.

\section{REFERÊNCIAS}

ALBANEZ, A. C. M. P. Caracterização dos fragmentos florestais a partir de estudos de ecologia da paisagem para o município de Ponte Nova, Minas Gerais. 2000. 160 f. Dissertação (Mestrado em Ciência Florestal) - Universidade Federal de Viçosa, Viçosa, 2000.

\section{ALMEIDA, C. G. Análise espacial dos fragmentos florestais na área do Parque Nacional dos Campos Gerais, Paraná. 2008. 72 f. Dissertação (Mestrado em Gestão do Território) - Universidade Estadual de Ponta Grossa, Ponta Grossa, 2008.}

\section{AMARAL, M.V.F. Dinâmica da estrutura da paisagem e mapeamento dos estádios sucessionais de reservas de floresta nativa, em um projeto de produção florestal. 2007. 130 f. Dissertação (Mestrado em Ciência Florestal) - Universidade Federal de Viçosa, Viçosa, 2007.}

AWADE, M.; METZGER, J. P. Importance of functional connectivity to evaluate the effect of hábitat fragmentation for three Atlantic rainforests birds. Austral Ecology, n. 33, p. 863-871, 2008.

\author{
BASILE, A. Caracterização estrutural e \\ física de fragmentos florestais no \\ contexto da paisagem da Bacia do Rio \\ Corumbataí, SP. 2006. 86 p. Dissertação \\ (Mestrado em Ecologia Aplicada) - Universidade \\ de São Paulo, Piracicaba, 2006.
}


BORGES, L.F.R.; SCOLFORO, J.R.; OLIVEIRA, A.D. et al. Inventário de fragmentos florestais nativos e propostas para seu manejo e o da paisagem. Cerne, v. 10, n. 1, p. 22-38, 2004.

BRITO, E.R.; MARTINS, S.V.; GLERIANI, J.M.; SOARES, V.P. Identification of degraded areas and classes of vegetal cover through geographical information system, for environmental adequacy. In: RODRIGUES, R.R.; MARTINS, S.V.; GANDOLFI, S. (Eds.). High diversity forest restoration in degraded areas: methods and projects in Brazil. New York: Nova Science Publishers, 2007. p.247-260.

COSTA, R.B. Fragmentação florestal e alternativas de desenvolvimento rural na região Centro-Oeste. Campo Grande: UCDB, 2003. 246 p.

FUSHITA, A. T. Análise da fragmentação de áreas de vegetação natural e seminatural do município de Santa Cruz da Conceição, São Paulo, Brasil. 2006. 84 f. Dissertação (Mestrado em Ecologia e Recursos Naturais). Universidade Federal de São Carlos, São Carlos, 2006.

\section{GOOGLE EARTH. Satellite's Image}

Software: versão 4.3 (beta). 2008. Disponível em <http://earth.google.com/>. Acesso em: 06 set. 2008.

GREGGIO, T.C.; PISSARRA, T.C.T.; RODRIGUES, F.M. Avaliação dos fragmentos florestais do município de Jaboticabal - SP. Revista Árvore, v.33, n.1, p.117-124, 2009.

MCGARIGAL, K., CUSHMAN, S.A.; NEEL, M.C.; ENE, E. Fragstats: Spatial pattern analysis program for categorical maps - version 3.3 build 5 Manual do programa. Computer software program produced by the authors at the University of Massachusetts, Amherst, 2002. Disponível em: $<$ www.umass.edu/landeco/research/fragstats/ fragstats.html>. Acesso em: 05 julho 2008.

NASCIMENTO, M.C.; SOARES, V.P.; RIBEIRO, C.A.Á.S.; SILVA, E. Mapeamento dos fragmentos de vegetação florestal nativa da Bacia hidrográfica do rio Alegre, Espírito Santo, a partir de imagens do Satélite IKONOS II. Revista Árvore, v.30, n.3, p.389-398, 2006.
OLIVEIRA, F.S.; SOARES, V.P.; PEZZOPANE, J.E.M. et al. Identificação de conflito de uso da terra em áreas de preservação permanente no entorno do Parque Nacional do Caparaó, estado de Minas Gerais. Revista Árvore, v.32, n.5, p.899-908, 2008.

PEREIRA, J.L.G.; BATISTA, G.T.; THALÊS, M.C.; ROBERTS, D.A.; VENTURIERI, A. Métricas da paisagem na caracterização da evolução da ocupação da Amazônia. Geografia, v.26, n. 1, 2001.

PÉRICO, E.; CEMIN, G. Caracterização da paisagem do município de Arvorezinha, RS, com ênfase na dinâmica dos fragmentos florestais, por meio de sistemas de informações geográficas (SIGs).

Scientia Forestalis, n. 70, p. 09-21, 2006.

RANTA, P.; BLOM, T.; NIEMELÄ, J.; JOENSUU, E.; SIITONEN, M. The fragmented Atlantic rain forest of Brasil: size, shape and distribution of forest fragments. Biodiversity and Conservation, v.7, p.385-403, 1998.

TURNER, M.G.; O’NEILL, R.V.; GARDNER, R.H.; MILNE, B.T. Effects of changing spatial scale on the analysis of landscape pattern. Landscape Ecology, v.3, n.3-4, p.153-162, 1989.

TONIAL, T. M. Dinâmica da paisagem da região nordeste do Estado do Rio Grande do Sul. 2003. 311 f. Tese (Doutorado em Ecologia e Recursos Naturais) Universidade Federal de São Carlos, São Carlos, 2003.

VIANA, V.M. Biologia e manejo de fragmentos florestais naturais. In: CONGRESSO FLORESTAL BRASILEIRO, 6., 1990, Campos do Jordão.

Anais... Campos do Jordão: SBS/SBEF, 1990, p. 113-118. Trabalho convidado.

WALDHOFF, P.; VIANA, V.M. Efeito de borda em um fragmento de mata atlântica em Linhares, ES. In: CONGRESSO FLORESTAL PANAMERICANO, 10.; CONGRESSO FLORESTAL BRASILEIRO, 70., 1993, Curitiba. Anais... Curitiba, 1993. v. 2, p. 41-44.

WICKHAM, J.D.; RIITTERS, K.H. Sensitivity of landscape metrics to pixel size. International Journal of Remote Sensing, v.16, n.18, p.3585-3594, 1995. 